\title{
Comportamiento del infarto agudo de miocardio en una unidad de cuidado intensivo de una ciudad intermedia durante un año
}

\section{Behavior of acute myocardial infarctions in an intensive care unit of a medium-size city over a one-year span}

\author{
Juan David Lobo-Hernández, Gilberto García-Zuluaga, Olga Alicia Nieto-Cárdenas \\ - Armenia (Colombia)
}

DOI: https://doi.org/10.36104/amc.2020.1498

\section{Resumen}

Identificar el comportamiento clínico y paraclínico del infarto de miocardio en una unidad de cuidados intensivos de una ciudad intermedia durante el 2017.

Métodos: estudio descriptivo de corte transversal, incluyó 99 registros con diagnóstico de infarto del miocardio al egreso de UCI. Se describieron las variables y se realizaron análisis de varianza y chi $^{2}$ y se consideró un valor de $\mathrm{p}<0.05$ para diferencias estadísticamente significativas.

Resultados: la prevalencia de infarto del miocardio fue $11.8 \%$ en UCI. La edad promedio fue 66.8 años. El dolor precordial estuvo presente en $82.8 \%$ con una duración promedio de 335 minutos. La irradiación del dolor fue más frecuente a miembro superior izquierdo (13.1\%). El infarto sin elevación del complejo ST fue más frecuente $(50.5 \%)$. Y la localización electrocardiográfica fue en la cara inferior en $28.2 \%$. El promedio de troponina I ultrasensible fue de $28.2 \mathrm{ng} / \mathrm{mL}$. Presentaron complicaciones el 54.5\%. La mortalidad fue de $14.1 \%$. La estancia hospitalaria fue 6.5 y la estancia en cuidados críticos tres días. Se encontró relación estadísticamente significativa entre dolor precordial y elevación del segmento ST $(\mathrm{p}<0.001)$ y entre troponina I con estancia hospitalaria y elevación del segmento $\mathrm{ST}$ ( $\mathrm{p}=0.007$ y p=0.003 respectivamente).

Conclusión: se identificó que $11.8 \%$ de los pacientes que ingresan a la UCI, lo hacen por infarto del miocardio, y una relación estadísticamente significativa entre dolor precordial con elevación del segmento ST y entre el aumento de troponina I con mayor estancia hospitalaria y elevación del segmento ST. (Acta Med Colomb 2020; 45. DOI: https://doi.org/10.36104/amc.2020.1498).

Palabras clave: infarto del miocardio, cuidados críticos, electrocardiografía, troponina I.

\section{Abstract}

Objectives: to identify the clinical and paraclinical behavior of myocardial infarctions in an intensive care unit of a medium-size city during 2017.

Methods: a descriptive, cross-sectional study which included 99 medical records with a diagnosis of myocardial infarction on ICU discharge. The variables were described and analysis of variance and $\mathrm{Chi}^{2}$ tests were performed, using a p-value less than 0.05 for statistically significant differences.

Results: the prevalence of myocardial infarction in the ICU was $11.8 \%$. The average age was 66.8 years. Chest pain was present in $82.8 \%$ with an average duration of 335 minutes. The pain most often radiated to the left arm $(13.1 \%)$. Non-ST segment elevation myocardial infarction was the most frequent $(50.5 \%)$, and the electrocardiogram located the infarctions on the inferior surface in $28.2 \%$. The average ultrasensitive troponin I was $28.2 \mathrm{ng} / \mathrm{mL}$. A total of $54.5 \%$ had complications. Mortality was $14.1 \%$. Hospital stay was 6.5 and critical care stay was three days. There was a statistically significant relationship between chest pain and ST segment elevation $(\mathrm{p}<0.001)$ and between troponin I plus hospital stay and ST segment elevation $(\mathrm{p}=0.007$ and $\mathrm{p}=0.003$, respectively).
Dr. Juan David Lobo-Hernández: Médico Interno. Grupo de Investigación Salud Pública, Programa de Medicina; Dr. Gilberto GarcíaZuluaga: Médico. Especialista en Cuidado Crítico; Dra. Olga Alicia Nieto Cárdenas: MD, MPH, PhD. Grupo de Investigación Salud Pública, Programa de Medicina. Facultad de Ciencias de la Salud, Universidad del Quindío. Armenia (Colombia).

Correspondencia: Dra. Olga Alicia NietoCárdenas. Armenia (Colombia).

E-mail: olgalicianieto@gmail.com Recibido: 30/VIII/2019 Aceptado: 11/III/2020 
Conclusion: the study showed that $11.8 \%$ of patients admitted to ICU are admitted for myocardial infarction, and there is a statistically significant relationship between chest pain and an elevated ST segment and between increased troponin I plus a longer hospital stay and ST segment elevation.

(Acta Med Colomb 2020; 45. DOI: https://doi.org/10.36104/amc.2020.1498).

Key words: myocardial infarction, critical care, electrocardiography, troponin.

\section{Introducción}

La cuarta definición clínica de infarto agudo del miocardio (IAM), según el Consenso de la Sociedad Europea de Cardiología (ESC 2018), se basa en la presencia de daño miocárdico agudo detectado por la elevación de biomarcadores cardiacos en el contexto de evidencia de isquemia miocárdica (1).

El infarto del miocardio se encuentra dentro de las 10 principales causas de mortalidad en el mundo (2). Esto coincide con las cifras para Colombia reveladas por el ASIS (Análisis de situación de salud, 2016), donde las enfermedades del sistema cardiovascular fueron la primera causa de mortalidad y la enfermedad isquémica cardiaca representó $49.3 \%$ del total de estos decesos (3).

Y representa un costo alto en el sistema de salud colombiano, que para el 2014 fue $\$ 13883087744$ es decir, el $7.2 \%$ del presupuesto para la salud de ese año (4).

En el departamento del Quindío el análisis de la situación de salud identificó que en el grupo etario de 15-44 años, la enfermedad isquémica del corazón aparece como la quinta causa de muerte y lidera la lista para los grupos de 45-64 años, 65 años y más (5). La prevalencia de infarto agudo de miocardio en la ciudad de Armenia, según un estudio realizado en un programa de riesgo cardiovascular de una institución prestadora de servicios de salud fue $2.49 \%$ (6).

La evaluación diagnóstica de personas en las que se sospeche esta patología se realiza con tres recursos claves:

1. Cuadro clínico: dolor en área precordial y/o epigástrica de 20 minutos de evolución, que aparece en reposo o en ejercicio; puede irradiarse a áreas de mandíbula, miembro superior izquierdo y región dorsal (7).

2. Manifestaciones electrocardiográficas: se puede observar en el electrocardiograma (ECG) de 12 derivaciones, la elevación o depresión del segmento ST (evaluada en el punto J), las ondas T hiperagudas y la onda Q son indicadores electrocardiográficos de infarto agudo al miocardio (7).

3. Biomarcadores séricos de necrosis miocárdica: El diagnóstico actual se hace con troponina I ultrasensible, aunque se han utilizado otros marcadores tales como la creatina quinasa fracción miocárdica (CK-MB) y la mioglobina $(1,7)$.

Para fines diagnósticos y terapéuticos el infarto agudo de miocardio se clasifica según hallazgos electrocardiográficos en infarto agudo de miocardio con elevación del segmento ST (IAMCEST) y sin elevación del ST (IAMNOST); y por coronariografía se reconoce el MINOCA como infarto agu- do de miocardio sin enfermedad coronaria ateroesclerótica obstructiva (1).

En el IMCEST: la presentación de síntomas compatibles con isquemia miocárdica junto a la presencia de hallazgos electrocardiográficos compatibles con elevación del segmento $\mathrm{ST}$ al menos en dos derivaciones contiguas, son criterios suficientes para el diagnóstico de infarto y pese a que los biomarcadores están indicados en estos casos, no deben retrasar el inicio de la terapia de reperfusión $(8,9)$.

Según el consenso ESC 2018 (1), se reconoce una nueva clasificación de infarto en cinco tipos, según la cual, en el IM tipo 1 hay énfasis en la relación causal entre la rotura de placa y la aterotrombosis coronaria; IM tipo 2: se produce un desequilibrio entre el aporte y la demanda de oxígeno no relacionados con la aterotrombosis coronaria aguda; IM tipo 3: es una categoría útil para diferenciarlo de la muerte súbita cardiaca; IM tipos 4-5: énfasis en la diferenciación entre daño miocárdico relacionado con el procedimiento.

Para el tratamiento, la guía de práctica clínica propuesta por el Ministerio de Salud colombiano para la atención del síndrome coronario agudo, divide las intervenciones terapéuticas en tres momentos: atención inicial de urgencias y hospitalización, revascularización y prevención secundaria (9).

La identificación temprana del infarto del miocardio y la clasificación del riesgo, es relevante en términos del pronóstico, debido a que una intervención terapéutica temprana puede disminuir el riesgo de arritmias potencialmente mortales en la fase aguda a menos de $3 \%$, de las cuales la gran mayoría se presentan en las primeras 12 horas de cuadro clínico (7).

El manejo especializado de este cuadro clínico debe ser en una unidad de cuidados intermedios o intensivos con el objetivo fundamental de detectar a tiempo las arritmias cardiacas potencialmente mortales que puedan aparecer como complicación (10). El tiempo de reperfusión del paciente con IAM para limitar la extensión y la atención especializada en unidad de cuidados intensivos para el monitoreo y abordaje adecuado de las arritmias potencialmente mortales que se presentan como complicación de la enfermedad, son determinantes fundamentales en el pronóstico y calidad de vida de los pacientes (11).

Este estudio describe el comportamiento clínico y los biomarcadores del IAM en una UCI general de un tercer nivel de atención, en una ciudad intermedia, que se lleva a cabo por primera vez en esta población. 


\section{Material y métodos}

Estudio descriptivo de corte transversal, que fue aprobado por el Comité de Bioética de la Universidad del Quindío.

Población: se identificaron todos los registros de admisión a la unidad de cuidados intensivos de un hospital de tercer nivel de atención del departamento del Quindío durante el año 2017, y se tomaron para el presente estudio todos los que tuvieron diagnóstico de infarto de miocardio.

Criterios de inclusión y exclusión: pacientes mayores de 18 años admitidos a una unidad de cuidados intensivos del departamento del Quindío durante el periodo comprendido entre el $1^{\circ}$. de enero y 31 de diciembre de 2017 con diagnóstico de infarto del miocardio, el cual se encontró registrado en la historia clínica por el médico tratante e incluyó las variables que se definieron para el estudio: signos y síntomas, electrocardiograma y biomarcadores (troponina I ultrasensible y $\mathrm{CPK}$ ). No se incluyeron otras presentaciones clínicas del infarto, como los infartos perioperatorios, los que tenían síntomas no claros o en forma tardía.

Análisis de la información: se realizó la tabulación de la información en una base de datos en Excel. Se procesó y analizó mediante el software STATGRAPHICS Centurión, versión 17.2.04. Se describieron las variables cuantitativas con promedio, desviación estándar e intervalos de confianza (IC95\%). Las variables cualitativas se describieron con la frecuencia de presentación por categorías.

La comparación de las variables se realizó con un análisis de varianza (ANOVA) para las variables cuantitativas y una prueba de chi ${ }^{2}$ para las variables cualitativas; se consideró una diferencia estadísticamente significativa cuando el valor de $\mathrm{p}$ fue $<$ de 0.05 .

\section{Resultados}

Se identificaron 836 registros de admisiones a la unidad de cuidados intensivos de un hospital de tercer nivel de atención del departamento durante el año 2017, de los cuales 111 ingresaron con diagnóstico de infarto del miocardio y 99 tuvieron diagnóstico de infarto del miocardio al egreso, identificando una prevalencia de IAM de $11.8 \%$ en la UCI.

En la Tabla 1 se observan las características generales. La edad promedio de los participantes de este estudio fue 66.8 años, se identificó que el infarto del miocardio fue más frecuente en hombres $65.6 \%$. El peso promedio en los participantes fue $66.1 \mathrm{Kg}$, la talla promedio de $161.7 \mathrm{~cm}$ y el índice de masa corporal fue $25.3 \mathrm{Kg} / \mathrm{m}^{2}$. La duración promedio del dolor fue 335.9 minutos desde su inició hasta la llegada a UCI, es decir 5.6 horas. La troponina I ultrasensible, al ingreso a urgencias, presentó un promedio de $28.2 \mathrm{ng} / \mathrm{L}$, la CPK 330.2 Ui/L y la CK-MB 48.73Ui/L. La estancia hospitalaria promedio fue 6.5 días, el tiempo de atención en unidad de cuidado intensivo 3.0 días y el tiempo de atención en urgencias 16 horas.

En la Tabla 2 se pueden observar las características clínicas del infarto en esta población. El dolor precordial se presentó en $82.8 \%$ de los participantes y el dolor epigás- trico en $14.1 \%$. La irradiación del dolor fue más frecuente a miembro superior izquierdo (13.1\%), seguido de región dorsal en $5.5 \%$, a la mandíbula en $4.0 \%$, a miembros superiores en $4.0 \%$ y estuvo ausente en el $42.4 \%$. Los síntomas concomitantes presentes fueron disnea en $48.4 \%$ de los participantes y diaforesis en $21.2 \%$. La localización electrocardiográfica más frecuente fue la cara inferior en $28.2 \%$; seguido de la cara anteroseptal $7 \%$, cara anterior $5 \%$ y cara anterior extensa $2 \%$. La onda T se halló alterada en el $27.2 \%$ de los participantes y la alteración más frecuente fue la onda $\mathrm{T}$ invertida en $23.23 \%$. Las complicaciones se identificaron en $54.5 \%$ de los participantes, siendo las más frecuentes la aparición de arritmias cardiacas (fibrilación auricular y bloqueo AV) en $16.1 \%$, seguido de insuficiencia mitral en $14.1 \%$, insuficiencia cardiaca en $11.1 \%$ y shock cardiogénico en 4\%. La mortalidad del infarto del miocardio en unidad de cuidados intensivos identificada en este estudio fue $14.1 \%$.

Se llevó a cabo un análisis de varianza para la comparación de variables cuantitativas y cualitativas y se identificó una relación estadísticamente significativa entre troponina I con elevación del complejo ST (valor de $\mathrm{p}=0.003$ ) y entre troponina I con estancia hospitalaria (valor de $\mathrm{p}=0.007$ ).

Se hizo una comparación entre variables categóricas y se identificó una relación estadísticamente significativa $\left(\mathrm{chi}^{2}\right)$, entre elevación del complejo ST con dolor precordial (valor de $\mathrm{p}<0.001)$ y entre complicaciones con desenlace en unidad de cuidados intensivos (valor de $\mathrm{p}<0.001$ ).

En la Tabla 3 se observa el manejo, la terapia antiplaquetaria fue administrada como terapia dual de ASA y clopidogrel en $94.9 \%$ de los participantes y como monoterapia de clopidogrel en $4 \%$. La revascularización se realizó con intervención coronaria percutánea más stent en $37.3 \%$; y se realizó fibrinólisis con tecnecteplase en $21.2 \%$. La terapia de anticoagulación se realizó con enoxaparina en $54.5 \%$ y con otras heparinas de bajo peso molecular en el $44.4 \%$.

\section{Discusión}

Se presenta la situación del IAM en una UCI de tercer nivel de complejidad en una ciudad intermedia, en la cual se encontró una relación estadísticamente significativa entre dolor precordial y elevación del segmento ST y entre troponina I con estancia hospitalaria y elevación del segmento ST; si bien estos resultados, coinciden con otros hallados en Colombia y otras latitudes, nos aporta un punto de referencia en un contexto del actual consenso que nos propone el reto de trascender en su definición epidemiológica, clínica y de biomarcadores (1) que servirá de referencia para estudios posteriores.

Se identificó una prevalencia de infarto del miocardio en los pacientes hospitalizados en la unidad de cuidado intensivo de un hospital de tercer nivel de atención de $11.84 \%$, es decir, de los 836 pacientes que consultaron a la unidad de cuidado intensivo, 99 tuvieron diagnóstico de infarto del miocardio; lo cual evidencia la necesidad de una 
Juan David Lobo-Hernández y cols.

Tabla 1. Características generales de la población (variables numéricas).

\begin{tabular}{|c|c|c|c|}
\hline Variable y unidad de medida & $\begin{array}{l}\text { Promedio y desviación estándar } \\
\text { n= } 99 \text { pacientes }\end{array}$ & $\begin{array}{c}\text { Intervalo de confianza } \\
\text { de } 95 \% \text { (IC) }\end{array}$ & $\begin{array}{l}\text { Valor de referencia } \\
\text { (VR) }\end{array}$ \\
\hline Edad en años & $66.80 \pm 11,41$ & $64.53-69.08$ & \\
\hline Peso en $\mathrm{Kg}$ & $66.10 \pm 12.28$ & $63.59-68.62$ & \\
\hline Talla en cm & $161.72 \pm 6.99$ & $160.24-163.20$ & \\
\hline $\mathrm{IMC} \mathrm{Kg} / \mathrm{m}^{\circ}$ & $25.34 \pm 4.41$ & $24.40-26.28$ & $18.5-24.9$ \\
\hline cTnI- ng/mL & $28.24 \pm 70.41$ & $13.41-43.08$ & $\leq 0.4 \mathrm{ng} / \mathrm{mL}$ \\
\hline $\mathrm{CPK}-\mathrm{Ui} / \mathrm{L}$ & $330.28 \pm 253.17$ & $118.62-541.94$ & $\begin{array}{l}\text { Hombres: } 200 \mathrm{Ui} / \mathrm{L} \\
\text { Mujeres: } 168 \mathrm{Ui} / \mathrm{L}\end{array}$ \\
\hline CK-MB - Ui/L & $48.73 \pm 33.70$ & $20.56-76.91$ & $0-24$ \\
\hline Creatinina en $\mathrm{mg} / \mathrm{dL}$ & $1.05 \pm 0.40$ & $0.97-1.13$ & $\begin{array}{l}\text { Hombres: } 0.80-1.30 \\
\text { Mujeres: } 0.60-1.00\end{array}$ \\
\hline RBC en $10 * 6$ millones/uL & $4.23 \pm 0.64$ & $4.09-4.37$ & $3.80-5.20$ \\
\hline $\mathrm{HB}$ en $\mathrm{g} / \mathrm{dL}$ & $12.80 \pm 2.25$ & $12.34-13.26$ & $\begin{array}{l}\text { Hombres: } 14.0-17.5 \\
\text { Mujeres: } 12.3-15.3\end{array}$ \\
\hline Porcentaje de HTC & $38.38 \pm 6.45$ & $37.06-39.69$ & $\begin{array}{l}\text { Hombres: } 42.0-52.0 \\
\text { Mujeres: } 37.0-47.0\end{array}$ \\
\hline $\begin{array}{l}\text { WBC } \\
10 * 3 \text { células/uL }\end{array}$ & $10.43 \pm 4.21$ & $9.56-11.30$ & $5.0-10.0$ \\
\hline PCR mg/dL & $4.87 \pm 3.88$ & $2,26-7.48$ & $\leq 0.3$ \\
\hline Glicemia mg/dL & $143.31 \pm 78.89$ & $121.34-165.27$ & $70-110$ \\
\hline Porcentaje de HbAlc & $7.62 \pm 2.37$ & $6.24-8.99$ & $\leq 5.6$ \\
\hline Potasio sérico $\mathrm{mEq} / \mathrm{L}$ & $3.99 \pm 0.55$ & $3.87-4.11$ & $3.5-5$ \\
\hline Sodio sérico $\mathrm{mEq} / \mathrm{L}$ & $138.33 \pm 4.27$ & $137.42-139.23$ & $135-145$ \\
\hline HDL mg/dL & $52.57 \pm 28.50$ & $34.46-70.68$ & $\begin{array}{l}\text { Hombres: } 35-55 \\
\text { Mujeres: } 45-65\end{array}$ \\
\hline $\mathrm{LDL} \mathrm{mg} / \mathrm{dL}$ & $92.56 \pm 57.20$ & $54.13-131$ & $0-100$ \\
\hline Colesterol mg/dL & $172.23 \pm 51.63$ & $137.55-206.92$ & $0-200$ \\
\hline Triglicéridos mg/dL & $119.66 \pm 69.50$ & $75.50-163.82$ & $\begin{array}{l}\text { Hombres: } 30-150 \\
\text { Mujeres: } 35-135\end{array}$ \\
\hline $\begin{array}{l}\text { Duración del dolor en minutos (desde } \\
\text { su inicio) }\end{array}$ & $335.92 \pm 272.16$ & $248.88-422.96$ & \\
\hline Tiempo de atención en urgencias en horas & $16.46 \pm 19.72$ & $12.53-20.39$ & \\
\hline $\begin{array}{l}\text { Tiempo de atención en UCI en días } \\
\text { cumplidos }\end{array}$ & $3.77 \pm 3.29$ & $3.12-4.43$ & \\
\hline Estancia hospitalaria en días cumplidos & $7.63 \pm 6.15$ & $6.40-8.86$ & \\
\hline
\end{tabular}

Tabla 2. Características clínicas del Infarto de miocardio (variables categóricas).

\begin{tabular}{|c|c|c|}
\hline Variable & Categorías & Porcentaje $n=99$ pacientes \\
\hline Sexo & $\begin{array}{l}\text { Femenino } \\
\text { Masculino }\end{array}$ & $\begin{array}{l}34.34 \\
65.66\end{array}$ \\
\hline Dolor precordial & $\begin{array}{l}\text { No registrado } \\
\text { no } \\
\text { si }\end{array}$ & $\begin{array}{c}3.03 \\
14.14 \\
82.83\end{array}$ \\
\hline Dolor epigástrico & $\begin{array}{l}\text { No registrado } \\
\text { no } \\
\text { si }\end{array}$ & $\begin{array}{l}11.11 \\
74.75 \\
14.14 \\
\end{array}$ \\
\hline Irradiación del dolor & $\begin{array}{l}\text { Ausente } \\
\text { Cuello } \\
\text { Mandíbula } \\
\text { Miembro superior izquierdo } \\
\text { Miembro superior izquierdo y cuello } \\
\text { Miembro superior izquierdo y mandíbula } \\
\text { Miembro superior izquierdo y región dorsal } \\
\text { Miembros superiores } \\
\text { No registrado } \\
\text { Región dorsal } \\
\text { Región dorsal y cuello }\end{array}$ & $\begin{array}{c}42.42 \\
3.03 \\
4.04 \\
13.13 \\
1.01 \\
2.02 \\
4.04 \\
4.04 \\
20.20 \\
5.05 \\
1.01\end{array}$ \\
\hline
\end{tabular}

Continúa... página siguiente 
Continuación... Tabla 2. Características clínicas del Infarto de miocardio (variables categóricas).

\begin{tabular}{|c|c|c|}
\hline Variable & Categorías & Porcentaje $n=99$ pacientes \\
\hline Disnea & $\begin{array}{l}\text { No registrado } \\
\text { no } \\
\text { si }\end{array}$ & $\begin{array}{l}19.19 \\
32.32 \\
48.48\end{array}$ \\
\hline Diaforesis & $\begin{array}{l}\text { No registrado } \\
\text { no } \\
\text { si }\end{array}$ & $\begin{array}{l}23.23 \\
55.56 \\
21.21\end{array}$ \\
\hline Electrocardiograma & $\begin{array}{l}\text { No registrado } \\
\text { si }\end{array}$ & $\begin{array}{c}2.02 \\
97.98\end{array}$ \\
\hline Elevación del complejo ST & $\begin{array}{l}\text { No registrado } \\
\text { no } \\
\text { si }\end{array}$ & $\begin{array}{c}2.02 \\
50.51 \\
47.47\end{array}$ \\
\hline Localización del infarto por EKG & $\begin{array}{l}2 \text { o más localizaciones comprometidas } \\
\text { Anterior (V3 a V4) } \\
\text { Anterior extenso (V1 a V6) } \\
\text { Antero lateral (V1-V6, DI y aVL) } \\
\text { Antero septal (V1 a V4) } \\
\text { Inferior (DII, DIII y AVF) } \\
\text { Lateral (DI, AVL, V5 y V6) } \\
\text { No elevación del complejo ST } \\
\text { No registrado } \\
\text { Septal (V1 y V2) }\end{array}$ & $\begin{array}{c}1.01 \\
5.05 \\
2.02 \\
1.01 \\
7.07 \\
28.28 \\
2.02 \\
49.49 \\
3.03 \\
1.01\end{array}$ \\
\hline Alteración de la onda $\mathrm{T}$ & $\begin{array}{l}\text { No registrado } \\
\text { no } \\
\text { si }\end{array}$ & $\begin{array}{l}33.33 \\
39.39 \\
27.27\end{array}$ \\
\hline Categorías de Alteración de la onda T & $\begin{array}{l}2 \text { alteraciones de onda } \mathrm{T} \\
\text { No alteración de la onda } \mathrm{T} \\
\text { No registrado } \\
\text { Onda T asimétrica } \\
\text { Onda T hiperaguda } \\
\text { Onda T invertida }\end{array}$ & $\begin{array}{c}1.01 \\
39.39 \\
34.34 \\
1.01 \\
1.01 \\
23.23\end{array}$ \\
\hline Presencia de onda Q & $\begin{array}{l}\text { No registrado } \\
\text { no } \\
\text { si }\end{array}$ & $\begin{array}{c}42.42 \\
51.52 \\
6.06\end{array}$ \\
\hline Comorbilidades & $\begin{array}{l}2 \text { comorbilidades } \\
3 \text { o más comorbilidades } \\
\text { Anemia } \\
\text { Cardiopatía isquémica } \\
\text { Cor pulmonale } \\
\text { Diabetes mellitus } \\
\text { EPOC } \\
\text { Falla cardíaca } \\
\text { Hipertensión arterial } \\
\text { No registrado } \\
\text { Obesidad } \\
\text { Sin comorbilidades }\end{array}$ & $\begin{array}{c}22.22 \\
19.19 \\
1.01 \\
3.03 \\
1.01 \\
1.01 \\
1.01 \\
4.04 \\
29.29 \\
1.01 \\
2.02 \\
15.15\end{array}$ \\
\hline Tabaquismo & $\begin{array}{l}\text { Antecedente de tabaquismo } \\
\text { No registrado } \\
\text { Sin antecedente } \\
\text { Tabaquismo activo }\end{array}$ & $\begin{array}{c}18.18 \\
63,64 \\
1.01 \\
17.17\end{array}$ \\
\hline Complicaciones & $\begin{array}{l}\text { No registrado } \\
\text { no } \\
\text { si }\end{array}$ & $\begin{array}{c}3.03 \\
42.42 \\
54.55\end{array}$ \\
\hline $\begin{array}{l}\text { Complicaciones por diagnóstico de historia } \\
\text { clínica }\end{array}$ & $\begin{array}{l}\text { Dos complicaciones } \\
\text { Tres o más complicaciones } \\
\text { Arritmias } \\
\text { Edema pulmonar } \\
\text { Insuficiencia mitral } \\
\text { Insuficiencia cardiaca } \\
\text { Insuficiencia cardiaca y arritmia } \\
\text { No registrado } \\
\text { Shock cardiogénico y arritmia } \\
\text { Shock cardiogénico } \\
\text { Sin complicaciones }\end{array}$ & $\begin{array}{c}1.01 \\
2.02 \\
16.16 \\
1.01 \\
14.14 \\
11.11 \\
2.02 \\
4.04 \\
2.02 \\
4.04 \\
42.42\end{array}$ \\
\hline Afiliación SGSSS & $\begin{array}{l}\text { Contributivo } \\
\text { No afiliado } \\
\text { Régimen especial } \\
\text { Subsidiado }\end{array}$ & $\begin{array}{c}11.11 \\
6.06 \\
1.01 \\
81.82\end{array}$ \\
\hline
\end{tabular}


Tabla 3. Características relacionadas con el manejo.

\begin{tabular}{|c|c|c|}
\hline Variable & Manejo & Porcentaje \\
\hline Terapia anti plaquetaria (TPL) & $\begin{array}{l}\text { ASA + clopidogrel } \\
\text { Clopidogrel } \\
\text { No registrado }\end{array}$ & $\begin{array}{c}94.95 \\
4.04 \\
1.01\end{array}$ \\
\hline TPL dosis & $\begin{array}{l}\text { ASA } 100 \mathrm{mg} 1 \text { diaria. clopidogrel } 75 \mathrm{mg} 1 \text { diaria. } \\
\text { ASA } 100 \mathrm{mg} 3 \text { tabletas ahora y luego } 1 \text { diaria. clopidogrel } 75 \mathrm{mg} 3 \text { tabletas ahora y luego } 1 \text { diaria. } \\
\text { ASA } 100 \mathrm{mg} 3 \text { tableta ahora y luego } 1 \text { diaria. clopidogrel } 75 \mathrm{mg} 4 \text { tableta ahora y luego una diaria. } \\
\text { Clopidogrel } 75 \mathrm{mg} 1 \text { diaria. } \\
\text { Clopidogrel } 75 \mathrm{mg} 4 \text { tableta ahora y luego } 1 \text { diaria. } \\
\text { No registrado }\end{array}$ & $\begin{array}{c}59.60 \\
1.01 \\
30.30 \\
\\
3.03 \\
5.05 \\
1.01\end{array}$ \\
\hline Revascularización & $\begin{array}{l}\text { CABAG } \\
\text { Fibrinólisis - Alteplase } \\
\text { Fibrinólisis - Tecnecteplase } \\
\text { ICP } \\
\text { ICP + stent } \\
\text { No realizado } \\
\text { No registrado }\end{array}$ & $\begin{array}{c}1.01 \\
2.02 \\
21.21 \\
14.14 \\
37.37 \\
21.21 \\
3.03\end{array}$ \\
\hline Terapia de anticoagulación (TAC) & $\begin{array}{l}\text { Enoxaparina } \\
\text { Otras heparinas de bajo peso molecular } \\
\text { No indicado }\end{array}$ & $\begin{array}{c}54.55 \\
44.44 \\
1.01\end{array}$ \\
\hline Terapia inhibición de SRAA (TSRA) & $\begin{array}{l}\text { Dos medicamentos } \\
\text { ARAII } \\
\text { IECA } \\
\text { No indicado } \\
\text { No registrado }\end{array}$ & $\begin{array}{c}1.01 \\
36.36 \\
44,44 \\
13.13 \\
5.05\end{array}$ \\
\hline Terapia hipolipemiante (THL) & $\begin{array}{l}\text { Atorvastatina } \\
\text { No indicado }\end{array}$ & $\begin{array}{c}95.96 \\
4.04\end{array}$ \\
\hline Protección gástrica (PG) & $\begin{array}{l}\text { Antagonista de receptores } \mathrm{H} 2 \\
\text { Inhibidores de bomba de protones } \\
\text { No indicado } \\
\text { No registrado }\end{array}$ & $\begin{array}{l}67.68 \\
18.18 \\
12.12 \\
2.02\end{array}$ \\
\hline Betabloqueadores (BB) & $\begin{array}{l}\text { Carvedilol } \\
\text { Metoprolol } \\
\text { No indicado } \\
\text { No registrado }\end{array}$ & $\begin{array}{c}71.72 \\
12.12 \\
13.13 \\
3.03\end{array}$ \\
\hline
\end{tabular}

unidad de cuidados coronarios. Actualmente no se tienen estudios previos de prevalencia de infarto del miocardio en una unidad de cuidados intensivos en el departamento, que permitan realizar una comparación. En un programa de riesgo cardiovascular de Armenia, Quindío en el primer nivel de atención, se encontró que la prevalencia fue $2.49 \%$ (6).

La edad promedio de los participantes de éste fue 66.8 años. En comparación, un estudio multicéntrico llamado TRAPID-AMI (High Sensitivity Cardiac Troponin T assay for Rapid Rule-out of Acute Myocardial Infarction) con 1282 participantes Mueller et al. (12), identificó que la edad promedio de participantes con presentación clínica compatible con infarto del miocardio fue 62 años, y la edad promedio de los participantes con diagnóstico final de infarto del miocardio fue de 69. Por otra parte, en el Reino Unido por Shah A. et al. (13) con 1171 participantes con diagnóstico de infarto del miocardio tipo 1 , se identificó una edad promedio de 68 años. Podría decirse que la edad promedio es similar a la encontrada en otros estudios.

Se identificó que el infarto del miocardio fue más frecuente en hombres $65.66 \%$, situación similar al comparar con González et al. (14), describió que 75.1\% de los participantes eran hombres y en México, Mazno y Castro (15), describieron el $83.3 \%$ de sexo masculino.
Presentaron dolor precordial en el presente estudio $82.83 \%$, similar a un estudio realizado en Bogotá, Colombia, por Sprockel et al. (16), en personas con diagnóstico de síndrome coronario agudo atendidos en el hospital San José, demostró un porcentaje de participantes con dolor precordial de $81.2 \%$, lo cual difiere de un artículo de revisión de la literatura realizado por Lu L. et al, donde describen que cerca de $64 \%$ de los pacientes con infarto del miocardio no experimenta dolor precordial, es decir, infarto del miocardio silente (17). Otro artículo de revisión de literatura realizado por Valensi et al., plantea que el porcentaje de personas que presentaron infarto del miocardio silente fue mayor en estudios de poblaciones jóvenes que en estudios de poblaciones con mayor edad (18).

El presente estudio identificó una relación estadísticamente significativa entre dolor precordial con elevación del segmento ST (valor de p <0.001). En el Reino Unido en 796 participantes compatibles con síndrome coronario agudo, Body et al. describieron que los participantes con infarto del miocardio con elevación del segmento ST presentaron una probabilidad significativamente mayor de experimentar dolor precordial con una duración del dolor superior a una hora, por otra parte, señalaron que la mediana de duración del dolor para participantes con infarto del miocardio con 
elevación del segmento ST fue de 120 minutos, por su parte, la duración del dolor del infarto del miocardio sin elevación del segmento ST fue de 90 minutos (19).

En este estudio la irradiación más frecuente fue a miembro superior izquierdo (13.1\%), la disnea se presentó en $48.4 \%$ y la diaforesis en $21.2 \%$. En un estudio realizado en Bogotá los porcentajes difieren: la irradiación más frecuente fue a miembro superior izquierdo con $27.1 \%$ de los participantes, la disnea estuvo presente en $51.8 \%$ y diaforesis en $54.1 \%$ (16).

En este estudio se identificó en el electrocardiograma que 50.5\% presentó infarto del miocardio sin elevación del complejo ST, mientras $47.4 \%$ de los participantes presentó elevación del segmento ST. Estos porcentajes son similares a otro estudio llamado FAST-MI (French Registry on Acute ST-elevation and non-ST-elevation Myocardial Infarction 2015) realizado en 204 centros de atención médica en Francia, el cual identificó que $51 \%$ de los participantes presentaron infarto del miocardio sin elevación del complejo ST (20). Lo anterior señala una prevalencia mayor de infarto del miocardio sin elevación del complejo ST.

La localización más frecuente del infarto del miocardio por electrocardiografía, en el presente estudio, fue la cara inferior con $28.2 \%$ del total de los participantes, posteriormente, se encontró la cara anteroseptal $(7 \%)$ y la anterior $(5 \%)$. En el estudio realizado en Bogotá comentado anteriormente, la localización más frecuente del infarto del miocardio en la electrocardiografía fue la cara inferior en $50.8 \%$ de los participantes, seguido por la cara anterior en $34.4 \%$ (16). La comparación de los estudios señala la localización inferior como la más frecuente y la anterior como aquélla que le sigue.

El nivel promedio de troponina I hallado en este estudio fue de $28.24 \mathrm{ng} / \mathrm{mL}$. En un estudio realizado en dos hospitales de Bogotá, Colombia, por Rojas et al. en participantes con diagnóstico de infarto del miocardio, la troponina I inicial promedio fue de $0.15 \mathrm{ng} / \mathrm{mL}$ (21). Por otra parte, Manfredonia et al, en Roma (Italia), identificaron un promedio de troponina I máxima de $13.4 \mathrm{ng} / \mathrm{mL}$, en los participantes con evidencia de enfermedad coronaria obstructiva significativa (22). Lo anterior sugiere que el promedio de troponina I encontrado fue alto en comparación con la troponina I de otros estudios.

Se encontró una relación estadísticamente significativa entre los niveles de troponina I y la elevación del complejo $\mathrm{ST}$ (valor de $\mathrm{p}=0.003$ ). En la Universidad de Nuevo México, Henrie et al. concluyeron que los participantes con infarto del miocardio con elevación del segmento ST tenían niveles de troponina I más altos (23). Lo cual concuerda con los hallazgos del presente estudio.

Se encontró una relación estadísticamente significativa entre los niveles de troponina I y la estancia hospitalaria (valor de $\mathrm{p}=0.007$ ). Lo anterior está de acuerdo con un Arboine-Aguirre et al, quien determinó que la estancia hospitalaria de los participantes con infarto del miocardio que presentaron niveles de troponina I más altos fue mayor (24).
La hipertensión arterial fue la comorbilidad más frecuente afectando al $29.2 \%$ de los participantes, el antecedente de tabaquismo y el tabaquismo activo sumaron $35.3 \%$ de los participantes, la obesidad estuvo presente en $2 \%$ de los participantes y la diabetes mellitus en $1 \%$. En un estudio multicéntrico realizado por McCord et al. (25), la población con diagnóstico de infarto del miocardio que participó en el estudio presentó la comorbilidad de hipertensión arterial en $73.9 \%$, seguido de historial de infarto del miocardio en $34 \%$ y diabetes mellitus II en $25.4 \%$ y el porcentaje de fumadores fue $66.2 \%$. En Armenia (Quindío) (6), en primer nivel de atención, el porcentaje de participantes con infarto del miocardio que presentaron hipertensión arterial fue $84.3 \%$, por otra parte el porcentaje de fumadores fue $12.9 \%$. Al comparar la hipertensión arterial es la comorbilidad más frecuente en el IAM.

Se identificaron complicaciones en $54.5 \%$ de los participantes y una relación estadísticamente significativa, entre complicaciones y desenlace en unidad de cuidados intensivos (valor de $\mathrm{p}<0.001$ ). Martínez y Ravelo en Cuba encontraron que en pacientes con infarto agudo del miocardio con elevación del complejo ST admitidos a unidad de cuidado intensivo, la aparición de arritmias fue de 14.7\%, las complicaciones mecánicas en $7.3 \%$ y la insuficiencia cardiaca en $12.8 \%$ (26). Estos hallazgos son similares a los encontrados en este estudio.

Se encontró una mortalidad por infarto del miocardio de $14.14 \%$, la cual fue mayor que en otros estudios, como el realizado en Cuba por Sánchez et al quien identificó una mortalidad de $12.5 \%$ de pacientes con infarto del miocardio hospitalizados en una unidad de cuidado crítico (27). En una unidad de cuidados intensivos en Venezuela por Moldes et al. también identificó una mortalidad de $12.5 \%$ (28). El estudio MASCARA (Manejo del síndrome coronario agudo. Registro Actualizado) realizado en 57 centros de atención en salud de España, identificó una media de mortalidad total intrahospitalaria de $5.7 \%$ (29).

Se identificó una estancia hospitalaria promedio de 6.5 días, un tiempo de atención en unidad de cuidado intensivo de tres días y un tiempo de atención en urgencias promedio de 16 horas. Un estudio multicéntrico realizado en Estados Unidos por Stolker et al. con 35806 participantes con diagnóstico de infarto del miocardio en el ingreso a 348 unidades de cuidado intensivo, identificó que el promedio de estancia hospitalaria de los participantes admitidos en las unidades de cuidado intensivo de bajo volumen de pacientes fue 6.9 días y el promedio de estancia hospitalaria de los participantes admitidos a unidades de cuidado intensivo de alto volumen de pacientes fue de cinco días (30). La estancia hospitalaria está relacionada con múltiples factores del paciente y del manejo incluida la reperfusión temprana (12).

\section{Conclusiones}

El presente estudio identificó una prevalencia de infarto del miocardio en unidad de cuidado intensivo de $11.8 \%$, y 
una edad promedio de 66.8 años. El infarto del miocardio fue más frecuente en hombres con $65.6 \%$. El dolor precordial estuvo presente en $82.8 \%$ de los participantes con una duración promedio del dolor de 5.5 horas. El promedio de troponina I fue $28.24 \mathrm{ng} / \mathrm{mL}$.

Se encontraron relaciones estadísticamente significativas entre dolor precordial y elevación del segmento ST; y entre troponina I con estancia hospitalaria y elevación del segmento ST.

\section{Agradecimientos}

Al Hospital Departamental Universitario del Quindío San Juan de Dios, de Armenia, Quindío.

\section{Referencias}

1. Kristian Thygesen K, Alpert JS, Jaffe AF, Chaitman BR, Bax JJ, Morrow DA, White HD. Consenso ESC 2018 sobre la cuarta definición universal del infarto de miocardio. Rev Esp Cardiol. 2019;72(1):72.e1-e27.

2. Organización mundial de la salud [Internet]. Las 10 principales causas de defunción. Ginebra. 2018 May 24 [citado el 6 Jun 2018] Recuperado a partir de: http://www.who.int/es/news-room/fact-sheets/detail/the-top-10-causes-of-death

3. Ministerio de Salud y protección social [Internet]. Análisis de Situación de Salud. Colombia. 2016 Nov [citado el 6 Jun 2018] Recuperado a partir de: https:// www.minsalud.gov.co/sites/rid/Lists/BibliotecaDigital/RIDE/VS/ED/PSP/asiscolombia-2016.pdf

4. Dávila FA, Barajas FD, Valenzuela E. Cost of Acute Myocardial Infarction in Colombia. Value health. 2014; 20: 853 -943.

5. Gobernación del Quindío [Internet]. Análisis de la situación de salud del departamento del Quindío. Colombia. 2012. [citado el 8 Jun 2018] Recuperado a partir de: https://www.minsalud.gov.co/plandecenal/mapa/Analisis-de-situacionde-salud-del-departamento-del-Quindio.pdf

6. Bedoya-Rios CA, Mendoza-Lozano JP, Nieto-Cárdenas OA. Prevalencia de infarto de miocardio en un programa de riesgo cardiovascular de una institución prestadora de salud en Armenia-Quindío. RCC. 2016; 23(6): 561-67.

7. Sociedad europea de cardiología. Guía ESC 2015 sobre el tratamiento de los síndromes coronarios agudos en pacientes con elevación del segmento ST. Rev Esp Cardiol. 2017; 70(12): 1082.e1-e61

8. Sociedad europea de cardiología. Guía ESC 2015 sobre el tratamiento de los síndromes coronarios agudos en pacientes sin elevación persistente del segmento ST. Revista Española de Cardiología. 2015, 68(12):1125;1-64.

9. Ministerio de Salud y protección social de Colombia [Internet]. Guía de práctica clínica para el síndrome coronario agudo. Colombia. 2017. [citado el 8 Jun 2018] Recuperado a partir de: http://gpc.minsalud.gov.co/gpc_sites/Repositorio/ Conv_500/GPC_s_coronario/gpc_s_coronario_profesionales.aspx

10. Newby LK. Reconciling Systems for Acute Myocardial Infarction Definition and Coding Classification. Clin Chem Acta. 2017; 63(1):24-26.

11. Palacios E, Castro MA. Epidemiología del infarto agudo de miocardio en la Unidad de terapia intensiva. Rev Asoc Mex Med Crit y Ter Int. 2002; 16(4): 113 -18 .

12. Mueller C, Giannitsis E, Christ M, Ordóñez-Llanos J, Defilippi C, McCord $\mathbf{J}$, et al. Multicenter Evaluation of a 0-Hour/1-Hour Algorithm in the Diagnosis of Myocardial Infarction With High-Sensitivity Cardiac Troponin T. Ann Emerg Med. 2016; 68(1): 76-87.

13. Shah A, McAllister D, Mills R, Ken K, Churchhouse A, Fleming K, et al. Sensitive Troponin Assay and the Classification of Myocardial Infarction. Am. J. Med. 2015; 128(5): 493-501.

14. González V, García N, Fernández E, García J, Rodríguez L. El infarto agudo de miocardio en la unidad de cuidados intensivos. Análisis de supervivencia. Enferm Intensiva. 2003; 14 (4): 156-160.

15. Manzo P, Castro A. Epidemiología del infarto agudo de miocardio en la Unidad de terapia intensiva. Rev Asoc Mex Med Crit Ter Int. 2002; 16 (4): 113-18.

16. Sprockel J, Diaztagle J, Filizzola V, Uribe L, Alfonso C. Descripción clínica y tratamiento de los pacientes con síndrome coronario agudo. Acta Med Colomb. 2014; 39(2): 124-130.

17. Lu L, Liu M, Sun R, Zheng Y, Zhang P. Myocardial Infarction: Symptoms and Treatments. Cell Biochem Biophys. 2015; 72: 865-67.

18. Valensi P, Lorgis L, Cottinb Y. Prevalence, incidence, predictive factors and prognosis of silent myocardial infarction: A review ofthe literature. Arch Cardiovasc Dis. 2011; 104: 178-88.

19. Body R, Carley S, Wibberley C, MacDowell G, Ferguson J, Mackway-Jones $\mathbf{K}$. The value of symptoms and signs in the emergent diagnosis of acute coronary syndromes. Resuscitation. 2010; 81(3): 281-86.

20. Belle L, Cayla G, Cottin Y, Coste P, Khalife K, Labèque J, et al. French Registry on Acute ST-elevation and non-ST-elevation Myocardial Infarction 2015 (FASTMI 2015). Design and baseline data. Arch Cardiovasc Dis. 2017; 110(6-7): 366-78.

21. Rojas M, Rodriguez D, Diaztaglec J, Sprockel C. Caracterización de pacientes con infarto agudo de miocardio sin enfermedad coronaria obstructiva. repert med cir. 2017; 26 (1):22-6.

22. Manfredonia L, Lanza G, Crudo F, Lamendola F, Graciani F, Villano A, et al. Diagnostic role of echocardiography in patients admitted to the emergency room with suspect no-ST-segment elevation acute myocardial infarction. Eur Rev Med Pharmacol Sci. 2019; 23: 826-32.

23. Henrie N, Harvell B, Ernst A, Weiss S, Oglesbee S, Sarangarm D. Comparison of QTc and Troponin Levels in ST Elevation MIs Compared with Non-ST Elevation MIs. South Med J. 2017; 110 (3): 210-16.

24. Arboine L, Galván J, Palacios J, Guzmán D. Valor pronóstico de la troponina I posintervencionismo coronario percutáneo electivo. Gac Med Mex. 2018; 154: $320-26$

25. McCord J, Aurora L, Lindahl B, Giannitsis E, Calle-Muller C, Nowak R, et al. Symptoms Predictive of Acute Myocardial Infarction in the Troponin Era: Analysis from the TRAPID-AMI Study. Crit Pathw Cardiol. 2019; 18 (1) 10-5.

26. Martínez G, Ravelo R. In-hospital complications of ST-segment elevation myocardial Infarction. Rev Cub Med Mil. 2016; 45 (3).

27. Sánchez E, Rodríguez V, Soto A, Abreu M. Comportamiento del infarto agudo de miocardio en un centro médico de diagnóstico integral. Rev Cub Med Int Emerg 2008; 7(4).

28. Moldes M, González M, Hernández M, Prado L, Pérez M. Comportamiento de infarto agudo de miocardio en Cuidados Intensivos. Centro Diagnóstico Integral Simón Bolívar. Venezuela. Rev Med Electrón. 2017; 39(1): 43-52.

29. Ferreira-Gonza lez I, Permanyer-Miralda G, Marrugat J, Heras M, Cuñat J, Civeira E, et al. Estudio MASCARA(Manejo del S1'ndrome Coronario Agudo. Registro Actualizado). Resultados globales. Rev Esp Cardiol. 2008; 61: 803-16.

30. Stolker J, Badawi O, Spertus J, Nasir A, Kennedy K, Harris I. Intensive care units with low versus high volume of myocardial infarction: clinical outcomes, resource utilization, and quality metrics. J Am Heart Assoc. 2015; 4 (6): e001225. 